\title{
Density of CD8+ lymphocytes in biopsy samples combined with the circulating lymphocyte ratio predicts pathologic complete response to chemoradiotherapy for rectal cancer
}

\author{
This article was published in the following Dove Press journal: \\ Cancer Management and Research \\ Number of times this article has been viewed
}

\author{
Binyi Xiaol,* \\ Jianhong Peng ${ }^{1, *}$ \\ Rongxin Zhangl,* \\ Jing $X u^{2}$ \\ Yongchun Wang ${ }^{2}$ \\ Yujing Fang ${ }^{1,2}$ \\ Junzhong Lin' \\ Zhizhong $\operatorname{Pan}^{\prime}$ \\ Xiaojun $\mathrm{Wu}^{\prime}$ \\ 'Department of Colorectal Surgery, \\ ${ }^{2}$ Department of Experimental \\ Research, State Key Laboratory \\ of Oncology in South China, \\ Collaborative Innovation Center \\ for Cancer Medicine, Sun Yat-sen \\ University Cancer Center, Guangzhou, \\ People's Republic of China \\ *These authors contributed equally to \\ this work
}

Correspondence: Xiaojun Wu;

Zhizhong Pan

Department of Colorectal Surgery,

State Key Laboratory of Oncology in

South China, Collaborative Innovation

Center for Cancer Medicine, Sun Yat-sen

University Cancer Center, 65I Dongfeng

Road East, Guangzhou 5I 0060, People's

Republic of China

Tel +86 2087343 I 24

Fax +862087343637

Email wuxj@sysucc.org.cn;

panzhzh@sysucc.org.cn
Objectives: The systemic status and local immune status, as determined by the neutrophillymphocyte ratio (NLR) or the lymphocyte ratio (LYMR) and tumor-infiltrating lymphocyte (TIL) count, respectively, have been suggested as predictors of the tumor response to neoadjuvant chemoradiotherapy (nCRT) in rectal cancer, although the utility of these measures remains controversial. We aimed to investigate the values of the LYMR, NLR and TIL count and their combinations (TIL-LYMR/TIL-NLR) in predicting pathologic complete response (pCR) after nCRT. Patients and methods: Pretreatment biopsy samples and data from the blood tests of 92 patients with rectal cancer who underwent curative resection after nCRT were retrospectively obtained. CD8+ TILs were immunostained using an antibody against CD8. The density of CD8+ TILs was recorded as the number of CD8+ T cells per square millimeter, and the results were classified as either "high" or "low". The LYMR and NLR were calculated using pretreatment blood test data and categorized into either "high" or "low" groups. TIL-LYMR was graded as "low," "mid" or "high" when neither, one or both the CD8+ TIL count and LYMR were "high," respectively. TIL-NLR was graded similarly. The associations between TILs and LYMR, NLR and their combinations (TIL-LYMR/TIL-NLR) were evaluated.

Results: pCR was significantly associated with a high LYMR, a low NLR and increased chemotherapy cycles $(P=0.039, P=0.043$ and $P=0.015$, respectively), but not with the CD $8+\mathrm{TIL}$ count or carcinoembryonic antigen (CEA) level ( $P=0.100$ and $P=0.590$, respectively). Additionally, $40 \%$ of patients with high LYMR and $40.7 \%$ with low NLR achieved pCR, whereas only $19.7 \%$ with low LYMR and $20.3 \%$ with high NLR did so. When the combinations were assessed, TIL-LYMR showed a positive correlation with pCR $(P=0.038)$, while no association between TIL-NLR and pCR was found $(P=0.916)$. In multivariate analysis, TIL-LYMR remained an independent predictor of $\mathrm{pCR}$ (odds ratio $[\mathrm{OR}]=1.833,95 \%$ confidence interval $[\mathrm{CI}]=1.069-3.142, P=0.028)$

Conclusion: High LYMR, low NLR and high TIL-LYMR at baseline are predictive of pCR to nCRT for patients with rectal cancer. These parameters may help identify pCR patients and provide additional information for therapeutic decision-making.

Keywords: chemoradiotherapy, rectal cancer, pathologic complete response, tumor-infiltrating lymphocytes, lymphocyte ratio, neutrophil-lymphocyte ratio

\section{Introduction}

Neoadjuvant chemoradiotherapy (nCRT) followed by surgery is the standard treatment for locally advanced rectal cancer, with the advantages of tumor downstaging and local 
control improvement. ${ }^{1}$ For patients achieving pathologic complete response ( $\mathrm{pCR}$ ) after nCRT, a strategy called "watch and wait" can be considered as a substitute for surgery. ${ }^{2}$ Such a strategy offers pCR patients a noninvasive treatment without compromising the long-term clinical outcome. ${ }^{3,4}$ Therefore, it is important to identify this group of patients before the initiation of treatment. Indeed, many clinicopathological and molecular factors have been suggested to be associated with pCR, although their utility in clinical practice remains controversial. ${ }^{5-8}$

Recently, it has been noted that the immune response plays a significant role in the antitumor effect of radiation. Tumor cell death is induced not only by the direct damage caused by radiation but also by the intratumoral immune reaction that follows. ${ }^{9-11} \mathrm{~A}$ high density of tumor-infiltrating lymphocytes (TILs; CD4+, CD8+) in the primary tumor has been suggested as a predictor of better tumor responses to nCRT. ${ }^{12}$ In addition to the localized immune reaction, the systemic immune status, as measured with the circulating lymphocyte ratio (LYMR) and neutrophil-lymphocyte ratio (NLR), has also shown the predictive value for tumor downstaging and survival. ${ }^{13,14}$ However, whether the combination of these two immune reactions shows better predictive value for the tumor response remains unknown.

Based on the above evidence, we hypothesized that the local immune status, as measured by the CD8+TIL count, and host immune status, as measured with the NLR and LYMR, could be used as predictors of the tumor response to nCRT. Therefore, the aim of this study was to explore the correlation of pretreatment local and systemic immune status values with $\mathrm{pCR}$ and evaluate the predictive value of their combination.

\section{Patients and methods}

\section{Patients}

From May 2006 to June 2014, a total of 92 patients with locally advanced rectal cancer who received nCRT followed by curative tumor excision were retrospectively included in this study. All patients provided pretreatment biopsy samples. None of the patients had overt metastatic disease at the beginning of the treatment. Pelvic magnetic resonance imaging or transanal ultrasound was applied to assess the primary tumor stage. All patients had stage T3/T4 disease and/or involved lymph node(s) before treatment. The total dose of radiation was 50 Gy given in 25 fractions, and four cycles of concomitant chemotherapy with capecitabine $\left(1,000 \mathrm{mg} / \mathrm{mm}^{2}\right.$ of body surface area, days 1-14) and oxaliplatin $\left(130 \mathrm{mg} / \mathrm{mm}^{2}\right.$ of body surface area, day 1) were scheduled, with one, two and one cycle given before, during and after nCRT, respectively.
The number of cycles received varied from 1 to 4, depending on patient tolerance. The study was performed with approval from the institutional research ethics committee of Sun Yat-sen University Cancer Center. Written consent forms notifying the use of specimens and publication of the results were obtained on admission. The raw data in this paper has been successfully uploaded and locked onto Research Data Deposit with a RDD number of RDDA2017000321.

\section{Immunological assessment of biopsy samples}

Pretreatment biopsy samples were obtained before the initiation of nCRT. TILs were assessed by immunohistochemical staining of slides from formalin-fixed, paraffin-embedded tumor blocks using mouse monoclonal antibodies against CD8 (1:100, ZA0508; ZSGB-BIO, Beijing, China). For each slide, three random fields were chosen for the enumeration of CD8+TIL within the area where tumor cells were considered to be most strongly stained. Then, an average was reached and considered as the number of CD8+ TIL per field. The density of TILs was defined as the number of positive CD8 lymphocytes per square millimeter and was then graded as either "high" or "low" (cutoff $=80 / \mathrm{mm}^{2}$ ). This cutoff value yielded a minimal " $P$-value" in the analysis of correlation with pCR and was thus applied.

\section{Evaluation of hematological factors}

Blood samples were taken within 2 weeks before nCRT at our center. None of the patient had evidence of infectious complications such as fever, chills and headache at the time of blood withdrawal. The data from regular blood tests were assessed, including counts of white blood cells (WBCs), lymphocytes and neutrophils. The NLR was calculated as the count of neutrophils divided by the count of lymphocytes, and the LYMR was calculated as the count of lymphocytes divided by the count of WBCs. An NLR $\geq 2.0$ and an LYMR $\geq 0.3$ were considered elevated (high) using the "minimal $P$ value" method. The LYMR combined with the CD8+ TIL count (TIL-LYMR) was then classified into three levels: low (both LYMR and density of CD8+ TILs were low), mid (either LYMR or density of CD8+ TILs was low) and high (both LYMR and density of CD8+ TILs were high). The combination of NLR with CD8+ TILs (CD8-NLR) was similarly grouped as low, mid or high.

\section{Grading of tumor regression after nCRT}

The tumor response to nCRT was reevaluated by two independent pathologists blinded to the initial reports according to the 
Mandard tumor regression grade (TRG) system ${ }^{15}$ as follows: Grade 1, complete response with absence of residual cancer and fibrosis extending through the wall; Grade 2, presence of residual tumor cells scattered through the area of fibrosis; Grade 3, increase in the number of residual cancer cells, with predominant fibrosis; Grade 4, residual cancer outgrowing the area of fibrosis and Grade 5, absence of regressive changes. Grade 1 was referred to as pCR, while Grades 2-5 were considered to represent non-pCR.

\section{Statistical analysis}

The correlation of pCR with clinicopathological parameters was analyzed using univariate analysis. Comparison of blood cell counts and ratios between different response groups was performed using a nonpaired $t$-test or MannWhitney $U$-test as appropriate. Multivariate analysis using logistic regression was performed to determine independent factors impacting the tumor response. Statistical analysis was performed using IBM SPSS Statistics for Windows, version 23.0 (IBM Corporation, Armonk, NY, USA). A two-sided $P$-value $<0.05$ was considered as statistically significant.

\section{Ethical approval}

All procedures performed in studies involving human participants were in accordance with the ethical standards of the institutional and/or national research committee and with the 1964 Helsinki declaration and its later amendments or comparable ethical standards.

\section{Informed consent}

Written informed consent was obtained from all individual participants included in the study.

\section{Results \\ Patient characteristics}

A total of 92 patients who underwent nCRT followed by curative resection were included in this analysis. Among them, $62(67.4 \%)$ were male and $30(32.6 \%)$ were female, with a median age of 56 years (range 25-79 years). All the patients received long-term radiotherapy $(50 \mathrm{~Gy} / 25)$ and concurrent chemotherapy with Xeloda $\left(1,000 \mathrm{mg} / \mathrm{mm}^{2}\right.$ of body surface area) and oxaliplatin $\left(130 \mathrm{mg} / \mathrm{mm}^{2}\right)$. The chemotherapy cycles received varied according to patient tolerance, with $41(44.6 \%), 31(33.7 \%), 18(19.6 \%)$ and $2(2.2 \%)$ patients receiving four, three, two and one cycle(s), respectively. Total mesorectal excision was performed within 6-8 weeks after radiation. According to the TRG system, pCR was achieved in $24(26.1 \%)$ patients.

\section{Correlation of $\mathrm{PCR}$ with clinicopathological characteristics}

As shown in Table 1, pCR was associated with the received number of cycles of preoperative chemotherapy. Patients who received three or four cycles of chemotherapy were more likely to achieve $\mathrm{pCR}$ than patients who received two cycles $(31.9 \%$ vs. $5 \%, P=0.015)$. There were no differences in gender, age, tumor location, pretreatment carcinoembryonic antigen (CEA) and CA19-9 levels, surgical procedure, tumor differentiation and clinical $\mathrm{T}$ stage or $\mathrm{N}$ stage between the $\mathrm{pCR}$ and non-pCR groups.

Regarding hematological factors, patients who achieved pCR showed a higher ratio of lymphocytes than those who

Table I Correlation of PCR with clinicopathological characteristics $(\mathrm{n}=92)$

\begin{tabular}{|c|c|c|c|}
\hline \multirow[t]{2}{*}{ Variables } & \multicolumn{2}{|c|}{ PCR, n (\%) } & \multirow[t]{2}{*}{$P$-value } \\
\hline & Yes & No & \\
\hline \multicolumn{4}{|l|}{ Gender } \\
\hline Male & $17(27.4)$ & $45(72.6)$ & 0.676 \\
\hline Female & $7(23.3)$ & $23(76.7)$ & \\
\hline \multicolumn{4}{|l|}{ Age, years } \\
\hline$<60$ & $18(29.5)$ & $43(70.5)$ & 0.294 \\
\hline$\geq 60$ & $6(19.4)$ & $25(80.6)$ & \\
\hline \multicolumn{4}{|l|}{ Tumor location } \\
\hline Middle-upper $(5-15 \mathrm{~cm})$ & $17(27.0)$ & $46(73.0)$ & 0.773 \\
\hline Low $(0-5 \mathrm{~cm})$ & $7(24.1)$ & $22(75.9)$ & \\
\hline \multicolumn{4}{|l|}{ Pre-CRT CEA $(\mathrm{ng} / \mathrm{mL})$} \\
\hline$\leq 5$ & $13(26.0)$ & $37(74.0)$ & 0.590 \\
\hline$>5$ & $8(2 I . I)$ & $30(78.9)$ & \\
\hline \multicolumn{4}{|l|}{ Pre-CRT CAI9-9 (ng/mL) } \\
\hline$\leq 35$ & $17(23.3)$ & $56(76.7)$ & 0.231 \\
\hline$>35$ & $7(36.8)$ & $12(63.2)$ & \\
\hline \multicolumn{4}{|l|}{ 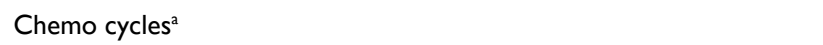 } \\
\hline $\mathrm{I}-2$ & I (5.0) & $19(95)$ & 0.015 \\
\hline $3-4$ & $23(31.9)$ & $49(68.1)$ & \\
\hline \multicolumn{4}{|l|}{ Interval (weeks) ${ }^{\mathrm{b}}$} \\
\hline $6-8$ & $15(25.4)$ & $44(74.6)$ & 0.846 \\
\hline$>8$ & $9(27.3)$ & $24(72.7)$ & \\
\hline \multicolumn{4}{|l|}{ Surgical procedure } \\
\hline$A R$ & I8 (27.7) & $47(72.3)$ & 0.586 \\
\hline APR or Hartmann & $6(22.2)$ & $21(77.8)$ & \\
\hline \multicolumn{4}{|l|}{ Tumor differentiation } \\
\hline Well & $6(42.9)$ & $8(57.1)$ & 0.262 \\
\hline Moderate & $13(21.7)$ & $47(78.3)$ & \\
\hline Poor & $5(27.8)$ & $13(72.2)$ & \\
\hline \multicolumn{4}{|l|}{ Pre-CRT T stage } \\
\hline 3 & $15(28.8)$ & 37 (7I.2) & 0.492 \\
\hline 4 & $9(22.5)$ & $31(77.5)$ & \\
\hline \multicolumn{4}{|l|}{ Pre-CRT N status } \\
\hline 0 & $9(31.0)$ & $20(69.0)$ & 0.463 \\
\hline I & $15(23.8)$ & $48(76.2)$ & \\
\hline
\end{tabular}

Notes: acycles of concurrent chemotherapy received. ${ }^{\mathrm{b}} \mathrm{Time}$ between surgery and last radiation.

Abbreviations: AR, anterior resection; ARP, abdominoperineal resection; $P C R$, pathologic complete response; CRT, chemoradiotherapy; CEA, carcinoembryonic antigen. 
did not ( 0.31 vs. $0.27, P=0.0031)$. However, the absolute counts of lymphocytes, WBCs, neutrophils, lymphocytes and the NLR were not significantly different between the two groups (Figure 1). When the NLR and LYMR were classified as "high" or "low," pCR was observed in more patients with high LYMR and TIL-LYMR and low NLR ( $P=0.039$, $P=0.038$ and $P=0.043$, respectively) (Table 2 and Figure 2).

CD8+ TILs were clearly immunostained in tumor nests using specific antibodies (Figure 2). Counts of CD8+ TILs in $\mathrm{pCR}$ patients were higher than those in non-pCR patients, and tumors with a high density of CD8+ TILs tended to achieve better regression than tumors with a low density, although statistical significance was not reached in either case ( $P=0.243$ and $P=0.10$, respectively). However, when CD8+ TILs were combined with the LYMR (TIL-LYMR), a significant difference in the $\mathrm{pCR}$ rate was observed among the different groups $(55.6 \%$ vs. $31.4 \%$ vs. $17.0 \%, P=0.038)$.

\section{Multivariate analysis of $\mathrm{pCR}$ predictors}

Variables with $P$-values $<0.1$ in the univariate analysis, which included CD8+ TILs, chemo cycles, NLR, LYMR and TILLYMR, were included in a multivariate analysis. To avoid colinearity, two similar multivariate analyses were performed separately, with one including CD8+ TILs, chemo cycles, NLR and LYMR, and the other including chemo cycles, NLR and TIL-LYMR. The results showed that when CD8+ TILs, chemo cycles, NLR and LYMR were analyzed, only chemo cycles remained significant (odds ratio $[\mathrm{OR}]=8.918,95 \%$ confidence interval $[\mathrm{CI}]=1.124-70.747, P=0.038$ ). However,

Table 2 Correlations of PCR with hematological and immunological factors $\left(n=92^{a}\right)$

\begin{tabular}{llll}
\hline Variables & pCR & P-value \\
\cline { 2 - 3 } & Yes & No & \\
\hline NLR & & & \\
$<2.0$ & $11(40.7)$ & $16(59.3)$ & 0.043 \\
$\geq 2.0$ & $13(20.3)$ & $51(79.7)$ & \\
LYMR & & & \\
$<0.3$ & $12(19.7)$ & $49(80.3)$ & 0.039 \\
$\geq 0.3$ & $12(40.0)$ & $18(60.0)$ & \\
CD8+ TIL & & & \\
Low & $15(21.7)$ & $54(78.3)$ & 0.100 \\
High & $9(39.1)$ & $14(60.9)$ & \\
TIL-NLR & & & \\
Low & $6(30.0)$ & $14(70.0)$ & 0.916 \\
Moderate & $14(25.5)$ & $41(74.5)$ & \\
High & $4(25.0)$ & $12(75.0)$ & \\
TIL-LYMR & & & \\
Low & $8(17.0)$ & $39(83.0)$ & 0.038 \\
Moderate & II (3I.4) & $24(68.6)$ & \\
High & $5(55.6)$ & $4(44.4)$ & \\
\hline
\end{tabular}

Note: ane patient's hematological data were not available.

Abbreviations: $\mathrm{pCR}$, pathologic complete response; NLR, neutrophil-lymphocyte ratio; LYMR, lymphocyte ratio; TIL, tumor-infiltrating lymphocyte; TIL-NLR, combination of CD8+ TIL and NLR; TIL-LYMR, combination of CD8+ TIL and LYMR.
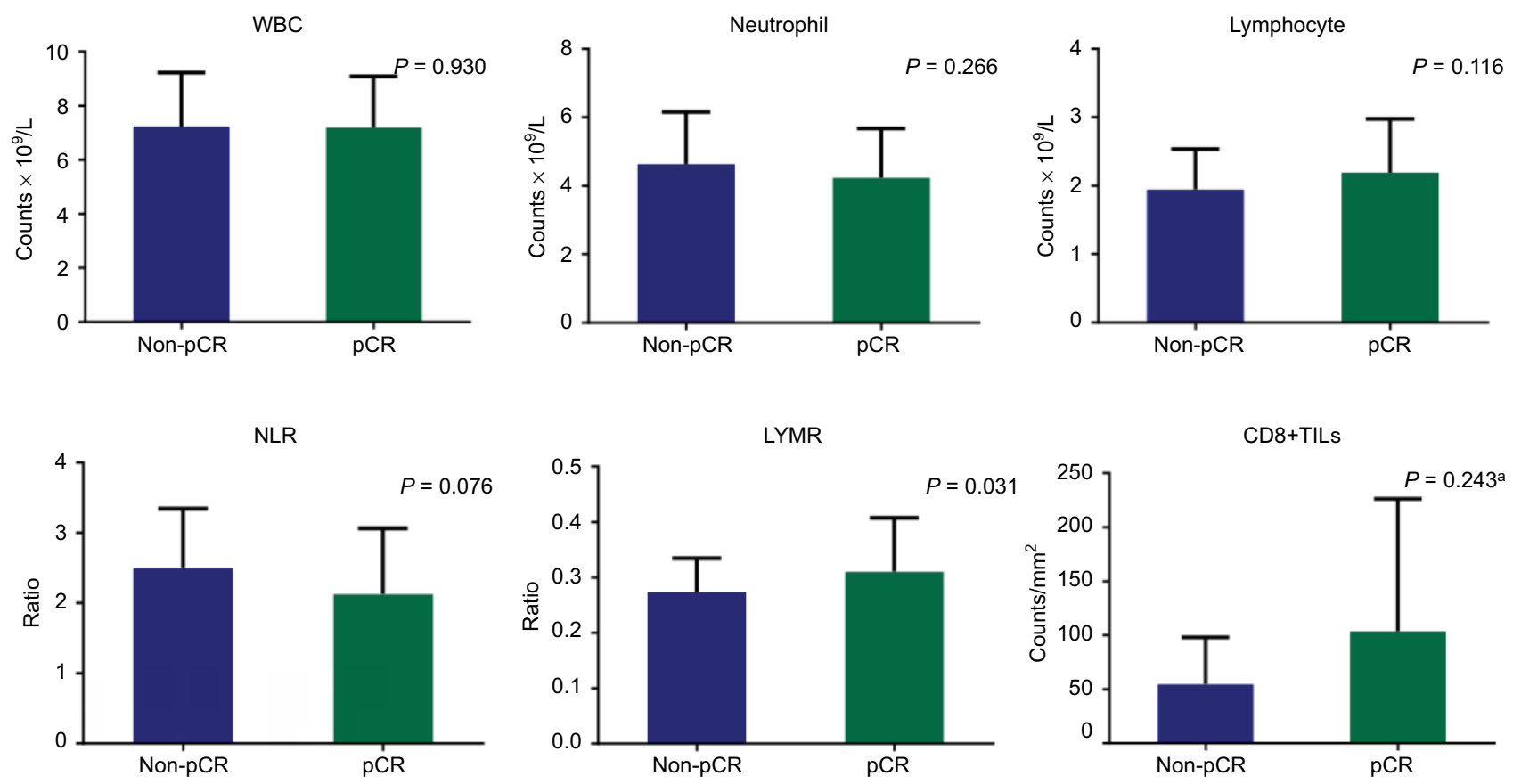

Figure I WBCs, neutrophils, lymphocytes, NLR, LYMR in circulating blood and CD8+ TIL counts in pretreatment biopsy samples taken before CRT in 68 non-pCR and 24 PCR cases.

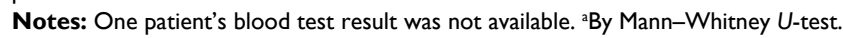

Abbreviations: WBC, white blood cell; NLR, neutrophil-lymphocyte ratio; LYMR, lymphocyte ratio; TIL, tumor-infiltrating lymphocyte; CRT, chemoradiotherapy; PCR, pathologic complete response. 
when chemo cycles, NLR and TIL-LYMR were analyzed, both chemo cycles and TIL-LYMR remained significant $(\mathrm{OR}=8.595,95 \% \mathrm{CI}=1.036-71.305, P=0.046$ and $\mathrm{OR}=1.833$, 95\% CI=1.069-3.142, $P=0.028$, respectively; Table 3).

\section{Correlation of LYMR/NLR with clinicopathologic parameters}

There was no correlation of LYMR or NLR with gender, age, CEA or CD8+ TILs $(P=0.370, P=0.918, P=0.878$ and $P=0.467$, respectively, and $P=0.353, P=0.698, P=0.616$ and $P=0.926$, respectively). Patients with a high LYMR and a low NLR tended to be more tolerant to chemotherapy, although statistical significance was not reached $(P=0.096$ and $P=0.057$, respectively; Table 4 ).

\section{Discussion}

In this study, we showed that the LYMR and NLR in circulating blood were associated with pCR after nCRT for rectal cancer and that the density of CD8+ TILs in biopsy samples combined with the circulating lymphocyte ratio (TIL-LYMR) was an independent predictor of pCR. Patients with a high density of CD8+ TILs and a high ratio of lymphocytes were more likely to achieve pCR. These results are generally
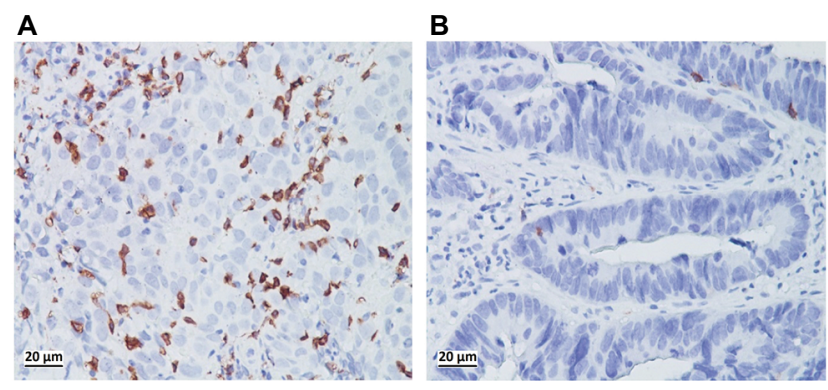

Figure 2 Immunostaining of CD8+ TILs in biopsy samples before CRT showed a high density $(\mathbf{A})$ and a low density $(\mathbf{B})$ of infiltration.

Abbreviations: TIL, tumor-infiltrating lymphocyte; CRT, chemoradiotherapy.

Table 3 Multivariate analyses for predictors associated with a PCR

\begin{tabular}{lll}
\hline Variables & OR $(95 \%$ Cl) & P-value \\
\hline $\begin{array}{l}\text { Not combined } \\
\quad \text { Chemo cycles }\end{array}$ & $8.918(1.124-70.747)$ & 0.038 \\
$\begin{array}{l}\text { Combined } \\
\text { Chemo cycles }\end{array}$ & $8.595(1.036-71.305)$ & 0.046 \\
TIL-LYMR & $1.833(1.069-3.142)$ & 0.028 \\
\hline
\end{tabular}

Notes: ${ }^{a}$ CD8 $8+$ TIL, chemo cycles, NLR and LYMR were analyzed. ${ }^{b}$ Chemo cycles, NLR and TIL-LYMR were analyzed.

Abbreviations: $P C R$, pathologic complete response; $O R$, odds ratio; $\mathrm{Cl}$, confidence interval; TIL-LYMR, combination of CD8+ TIL and LYMR; NLR, neutrophillymphocyte ratio; TIL, tumor-infiltrating lymphocyte; LYMR, lymphocyte ratio. consistent with those of previous studies, ${ }^{16-18}$ showing that the systemic status and local immune status are positively associated with the tumor response to nCRT. However, to our knowledge, our study is the first to evaluate the combination of the systemic status and local immune status for predicting the response to nCRT in rectal cancer.

The tumor microenvironment represents the front line of tumor-host interactions, and many inflammatory cells, immune cells and cytokines in this environment are involved in the process of tumor development. ${ }^{19-21}$ Among them, T lymphocytes (CD4+ and CD8+ lymphocytes) are generally considered to be antitumoral. Galon et al found that a high density of T cells in primary tumors was linked to improved clinical outcome and defined a scoring system called the immune score to predict survival based on lymphocyte infiltration in different sites of the tumor. ${ }^{22,23}$ In the nCRT setting, Yasuda et $\mathrm{al}^{12}$ studied the correlation of the density of CD4+ and CD8+ TILs in pre-chemoradiotherapy (CRT) biopsy samples with the tumor response and found that CD4+ and CD8+ TILs were strongly associated with tumor shrinkage. These findings revealed that intratumoral T-cell infiltration had a positive impact on radiosensitivity in rectal cancer. However, in our study, although a higher density of CD8+ TILs was observed in the pCR group than in the non-PCR group, the difference was not statistically significant (103.8 vs. 55.8, $P=0.243)$. A similar negative result was obtained when CD8+ TILs were analyzed by groups $(P=0.100)$. Given that previous studies in this field included relatively small sample sizes and did not follow uniform nCRT protocols, it is not surprising that different results have been reported.

The systemic immune status, as measured by the NLR and LYMR, is another factor affecting nCRT efficacy. An elevated NLR and a decreased LYMR before nCRT were suggested as predictors of poor tumor response in previous studies. ${ }^{24,13}$ In our study, the correlation of NLR and LYMR with pCR was also observed in the univariate analysis $(P=0.043$ and $P=0.039$, respectively), although this correlation failed to reach significance in the multivariate analysis. However, when LYMR was combined with CD8+ TILs, the new indicator, TIL-LYMR, showed a positive association with pCR $(P=0.038)$ and remained an independent factor in the multivariate analysis $(P=0.028)$. This finding suggests that the combination of the systemic status and local immune status might perform better than either status alone in predicting the tumor response to treatment.

The relationship between the systemic immune status and the tumor microenvironment has not been fully clarified. It was previously believed that circulating immune cells exerted 
Table 4 Correlation of LYMR/NLR with clinicopathological factors $(n=92)$

\begin{tabular}{|c|c|c|c|c|c|c|}
\hline \multirow[t]{2}{*}{ Variables } & \multicolumn{2}{|l|}{ LYMR } & \multirow[t]{2}{*}{$P$ value } & \multicolumn{2}{|l|}{ NLR } & \multirow[t]{2}{*}{$P$-value } \\
\hline & Low & High & & Low & High & \\
\hline \multicolumn{7}{|l|}{ Gender } \\
\hline Male & $39(63.9)$ & $22(36.1)$ & 0.37 & $20(32.8)$ & $41(67.2)$ & 0.353 \\
\hline Female & $22(73.3)$ & 8 (26.7) & & 7 (23.3) & $23(76.7)$ & \\
\hline \multicolumn{7}{|l|}{ Age (years) } \\
\hline$<60$ & $40(66.7)$ & $20(33.3)$ & 0.918 & $17(28.3)$ & 43 (7I.7) & 0.698 \\
\hline$\geq 60$ & $21(67.7)$ & $10(32.3)$ & & $10(32.3)$ & $21(67.7)$ & \\
\hline \multicolumn{7}{|l|}{ CD8+ TIL } \\
\hline High & $14(60.9)$ & $9(39.1)$ & 0.467 & $7(30.4)$ & $16(69.6)$ & 0.926 \\
\hline Low & 47 (69.I) & $21(30.9)$ & & $20(29.4)$ & $48(70.6)$ & \\
\hline \multicolumn{7}{|c|}{ Pre-CRT CEA } \\
\hline$>5$ & $25(67.6)$ & $12(32.4)$ & 0.878 & $10(27.0)$ & $27(73.0)$ & 0.616 \\
\hline$\leq 5$ & $33(66.0)$ & $17(34.0)$ & & $16(32.0)$ & $34(68.0)$ & \\
\hline \multicolumn{7}{|c|}{ Chemo cycles $^{a}$} \\
\hline $3-4$ & $44(62.0)$ & $27(38.0)$ & 0.096 & $25(35.2)$ & $46(64.8)$ & 0.057 \\
\hline $\mathrm{I}-2$ & $17(85.0)$ & $3(15.0)$ & & $2(10.0)$ & I8 (90.0) & \\
\hline
\end{tabular}

Note: a ${ }^{2}$ ycles of concurrent chemotherapy received.

Abbreviations: LYMR, lymphocyte ratio; NLR, neutrophil-lymphocyte ratio; TIL, tumor-infiltrating lymphocyte; CRT, chemoradiotherapy; CEA, carcinoembryonic antigen.

their antitumor effect by penetrating and infiltrating into the tumor environment. This assumption was supported by the study of Grimm et al, ${ }^{25}$ which evaluated circulating peripheral blood lymphocytes and TILs in tumor specimens of oral squamous cell carcinoma and found that circulating T-cell subsets (CD3+, CD4+ and CD8+) were significantly associated with their corresponding TIL subsets. In contrast, different results were derived in ovarian carcinoma, as Emerson el $\mathrm{al}^{26}$ suggested that intratumoral immune cells were distinct from those circulating in the blood and represented a population of $T$ cells specific to the tumor microenvironment. Here, we showed that both the LYMR and the NLR at baseline were not associated with CD8+ TILs in rectal cancer $(P=0.467$ and $P=0.926$, respectively). Patients with a high LYMR or a low NLR in the blood did not necessarily show dense infiltration of CD8+ TILs. Such inconsistency between the local and systemic immune reactions has also been observed in terms of the therapeutic response, as some studies have indicated that lymphocytes in the peripheral blood and the tumor nest respond differently to nCRT, with the circulating LYMR decreasing and the density of TILs increasing after nCRT. ${ }^{14,16}$

Other factors, such as the platelet count, serum albumin and CEA level, have been used to predict treatment responses in rectal cancer. ${ }^{27-29}$ In our study, we assessed the association between the CEA and CA 19-9 levels with pCR, but no statistical significance was found $(P=0.590$ and $P=0.231$, respectively). Patient compliance with concurrent chemotherapy should also be noted. In a Phase II trial conducted by Garcia-Aguilar et al, ${ }^{30}$ patients who received chemotherapy with mFOLFOX6 between chemoradiation and surgery achieved a higher rate of pCR than those who did not, and this rate grew as the cycles of chemotherapy received increased from two to six. In our study, all the patients received combined chemotherapy with oxaliplatin, and patients who received three to four cycles achieved a higher rate of $\mathrm{pCR}$ than those who received one to two cycles ( $46.9 \%$ vs. $5.3 \%$, $P=0.015)$. Multivariate analysis revealed that the number of preoperative chemotherapy cycles was an independent factor for pCR ( $P=0.038$ and $P=0.046$, respectively). Therefore, it is reasonable to assume that part of the predictive capacity of the NLR or LYMR, as reported in many studies, can be attributed to chemotherapy tolerance, as patients with a higher level of pre-CRT lymphocytes tend to tolerate more cycles of chemotherapy before surgery; however, in our study, statistical significance was not reached (61.4\% vs. $17.6 \%, P=0.096)$.

There are some limitations to this study. First, the sample size of our study was small, and many cutoff values yielded from the dataset are different from those of previous studies, which may compromise comparability. Second, circulating lymphocytes and neutrophils can be affected by many other conditions, such as infectious and cardiovascular diseases, ${ }^{31,32}$ which were not evaluated in this study. Additionally, our evaluation of CD8+ TILs was performed using biopsy samples from one site of the tumor, not from the center and margin of the tumor, as previous studies have done. ${ }^{17}$

\section{Conclusion}

Taken together, our study showed that the NLR, LYMR and combination represented by TIL-LYMR were predictive of pCR after chemoradiation for rectal cancer. TIL-LYMR, in particular, reflecting both the local and systemic immune reactions, may serve as an independent predictor of $\mathrm{pCR}$ 
and help to identify patients to receive "watch and wait" treatment instead of surgery. More studies with larger samples should be performed to confirm the value of this combined marker.

\section{Acknowledgments}

We thank professors Peirong Ding, Zhenhai Lu, Gong Chen and Liren Li for their generosity in providing study patients and offering insightful guidance for this study. A special thanks is due to Sherry Young, without whose unselfish support and encouragement this paper would never be completed. The study was funded by National Natural Science Foundation of China (No. 81502459 and No. 81772595), Science and Technology Project in Guangdong Province (No. 2013B021800146) and Sun Yat-sen University Clinical Research 5010 Program (No. 2014010).

\section{Disclosure}

The authors report no conflicts of interest in this work.

\section{References}

1. Bosset JF, Collette L, Calais G, et al; EORTC Radiotherapy Group Trial 22921. Chemotherapy with preoperative radiotherapy in rectal cancer. N Engl J Med. 2006;355(11):1114.

2. Habr-Gama A, Perez RO, Nadalin W, et al. Operative versus nonoperative treatment for stage 0 distal rectal cancer following chemoradiation therapy: long-term results. Ann Surg. 2004;240(4):717-718.

3. Lambregts DMJ, Maas M, Bakers FCH, et al. Long-term follow-up features on rectal MRI during a wait-and-see approach after a clinical complete response in patients with rectal cancer treated with chemoradiotherapy. Dis Colon Rectum. 2011;54(12):1521-1528.

4. Smith JD, Ruby JA, Goodman KA, et al. Nonoperative management of rectal cancer with complete clinical response after neoadjuvant therapy. Ann Surg. 2012;256(6):965-972.

5. Carlomagno C, Pepe S, D'Armiento FP, et al. Predictive factors of complete response to neoadjuvant chemoradiotherapy in patients with rectal cancer. Oncology. 2010;78(5-6):369-375.

6. Kalady MF, de Camposlobato LF, Stocchi L, et al. Predictive factors of pathologic complete response after neoadjuvant chemoradiation for rectal cancer. Ann Surg. 2009;250(4):582.

7. Zeng W, Liang J, Wang Z, et al. Clinical parameters predicting pathologic complete response following neoadjuvant chemoradiotherapy for rectal cancer. Chin J Cancer. 2015;34(10):468-474.

8. Jung SW, Park IJ, Oh SH, et al. Association of immunologic markers from complete blood counts with the response to preoperative chemoradiotherapy and prognosis in locally advanced rectal cancer. Oncotarget. 2017;8(35):59757-59765.

9. Frey B, RubnerY, Kulzer L, et al. Antitumor immune responses induced by ionizing irradiation and further immune stimulation. Cancer Immunol Immunother. 2014;63(1):29-36.

10. Lorimore SA, Coates PJ, Scobie GE, Milne G, Wright EG. Inflammatory-type responses after exposure to ionizing radiation in vivo: a mechanism for radiation-induced bystander effects? Oncogene. 2001;20(48):7085-7095.

11. Perez CA, Fu A, Onishko H, Hallahan DE, Geng L. Radiation induces an antitumour immune response to mouse melanoma. Int J Radiat Biol. 2009;85(12):1126-1136.
12. Yasuda K, Nirei T, Sunami E, Nagawa H, Kitayama J. Density of CD4(+) and CD8(+) T lymphocytes in biopsy samples can be a predictor of pathological response to chemoradiotherapy (CRT) for rectal cancer. Radiat Oncol. 2011;6(1):49.

13. Dou X, Wang R, Yan H, et al. Circulating lymphocytes as predictors of sensitivity to preoperative chemoradiotherapy in rectal cancer cases. Asian Pac J Cancer Prev. 2013;14(6):3881-3885.

14. Kitayama J, Yasuda K, Kawai K, Sunami E, Nagawa H. Circulating lymphocyte number has a positive association with tumor response in neoadjuvant chemoradiotherapy for advanced rectal cancer. Radiat Oncol. 2010; $5(1): 47$.

15. Mandard AM, Dalibard F, Mandard JC, et al. Pathologic assessment of tumor regression after preoperative chemoradiotherapy of esophageal carcinoma. Clinicopathologic correlations. Cancer. 1994;73(11):2680-2686.

16. Teng F, Mu D, Meng X, et al. Tumor infiltrating lymphocytes (TILs) before and after neoadjuvant chemoradiotherapy and its clinical utility for rectal cancer. Am J Cancer Res. 2015;5(6):2064-2074.

17. Galon J. Type, density, and location of immune cells within human colorectal tumors predict clinical outcome. Science. 2006;313(5795):1960-1964.

18. Malietzis G, Giacometti M, Askari A, et al. A preoperative neutrophil to lymphocyte ratio of 3 predicts disease-free survival after curative elective colorectal cancer surgery. Ann Surg. 2014;260(2):287-292.

19. Lewis CE, Pollard JW. Distinct role of macrophages in different tumor microenvironments. Cancer Res. 2006;66(2):605-612.

20. Houghton MG, Rzymkiewicz DM, Ji H, et al. Neutrophil elastasemediated degradation of IRS-1 accelerates lung tumor growth. Nat Med. 2010;16(2):219-223.

21. Seruga B, Zhang H, Bernstein LJ, et al. Cytokines and their relationship to the symptoms and outcome of cancer. Nat Rev Cancer. 2008;8(11):887-899.

22. Mlecnik B, Bindea G, Angell HK, et al. Integrative analyses of colorectal cancer show immunoscore is a stronger predictor of patient survival than microsatellite instability. Immunity. 2016;44(3):698-711.

23. Mlecnik B, Tosolini M, Kirilovsky A, et al. Histopathologic-based prognostic factors of colorectal cancers are associated with the state of the local immune reaction. J Clin Oncol. 2011;29(6):610-618.

24. Kim IY, You SH, Kim YW. Neutrophil-lymphocyte ratio predicts pathologic tumor response and survival after preoperative chemoradiation for rectal cancer. BMC Surg. 2014;14:94.

25. Grimm M, Feyen $\mathrm{O}$, Hofmann $\mathrm{H}$, et al. Immunophenotyping of patients with oral squamous cell carcinoma in peripheral blood and associated tumor tissue. Tumor Biol. 2016;37(3):3807-3816.

26. Emerson RO, Sherwood AM, Rieder MJ, et al. High-throughput sequencing of T-cell receptors reveals a homogeneous repertoire of tumour-infiltrating lymphocytes in ovarian cancer. J Pathol. 2013;231(4):433-440.

27. Park JW, Lim SB, Kim DY, et al. Carcinoembryonic antigen as a predictor of pathologic response and a prognostic factor in locally advanced rectal cancer patients treated with preoperative chemoradiotherapy and surgery. Int J Radiat Oncol Biol Phys. 2009;74(3):810-817.

28. Wan S, Lai Y, Myers RE, et al. Preoperative platelet count associates with survival and distant metastasis in surgically resected colorectal cancer patients. J Gastrointest Cancer. 2013;44(3):293-304.

29. Yasuda K, Sunami E, Kawai K, Nagawa H, Kitayama J. Laboratory blood data have a significant impact on tumor response and outcome in preoperative chemoradiotherapy for advanced rectal cancer. $J$ Gastrointest Cancer. 2012;43(2):236-243.

30. Garcia-Aguilar J, Chow OS, Smith DD, et al; Timing of Rectal Cancer Response to Chemoradiation Consortium. Effect of adding mFOLFOX6 after neoadjuvant chemoradiation in locally advanced rectal cancer: a multicentre, phase 2 trial. Lancet Oncol. 2015;16(8):957-966.

31. Farah R, Khamisy Farah R. Association of neutrophil to lymphocyte ratio with presence and severity of gastritis due to Helicobacter pylori infection. J Clin Lab Anal. 2014;28(3):219-223.

32. Bhat T, Teli S, Rijal J, et al. Neutrophil to lymphocyte ratio and cardiovascular diseases: a review. Expert Rev Cardiovasc Ther. 2013;11(1):55. 


\section{Publish your work in this journal}

Cancer Management and Research is an international, peer-reviewed open access journal focusing on cancer research and the optimal use of preventative and integrated treatment interventions to achieve improved outcomes, enhanced survival and quality of life for the cancer patient. The manuscript management system is completely online and includes a very quick and fair peer-review system, which is all easy to use. Visit http://www.dovepress.com/testimonials.php to read real quotes from published authors.

Submit your manuscript here: https://www.dovepress.com/cancer-management-and-research-journal 